\title{
MR features of primary and secondary malignant lymphoma of the pancreas: a pictorial review
}

\author{
Yasunari Fujinaga • Chandana Lall • Aashish Patel • \\ Tsuyoshi Matsushita $\cdot$ Rupan Sanyal • Masumi Kadoya
}

Received: 6 December 2012 /Revised: 14 February 2013 / Accepted: 22 February 2013 /Published online: 20 March 2013

(C) The Author(s) 2013. This article is published with open access at Springerlink.com

\begin{abstract}
Objective To describe the imaging findings of primary and secondary pancreatic malignant lymphoma on magnetic resonance imaging (MRI), to help differentiate lymphoma of the pancreas from primary adenocarcinoma and autoimmune pancreatitis among others, and to discuss a few atypical presentations of pancreatitis mimicking lymphoma.

Conclusion Knowledge of these imaging manifestations of lymphoma may be helpful to arrive at an accurate diagnosis and avoid unnecessary morbidity and mortality from inadvertent surgery.

Main Messages

- Pancreatic malignant lymphoma is shown as a nodular lowdensity area with mild enhancement on CT.

- It sometimes shows variable manifestations mimicking other tumours and inflammatory conditions.

- MRI provides useful information for differentiating malignant lymphoma from other mimickers.
\end{abstract}

Y. Fujinaga $(\bowtie) \cdot$ T. Matsushita $\cdot$ M. Kadoya

Department of Radiology, Shinshu University School of Medicine, 3-1-1 Asahi,

Matsumoto, 390-8621, Japan

e-mail: fujinaga@shinshu-u.ac.jp

Y. Fujinaga $\cdot$ C. Lall

Department of Radiological Sciences, University of California, Irvine Medical Center, 101 The City Drive South, Route 140, Orange, CA 92868, USA

A. Patel

Department of Radiology, Abdominal Imaging, Indiana University Medical Center, 550 N University Blvd,

Indianapolis, IN 46202, USA

R. Sanyal

Department of Diagnostic Radiology, Abdominal Imaging,

Cleveland Clinic Hospitals, 9500 Euclid Ave.,

Cleveland, OH 44195, USA
Keywords Magnetic resonance imaging · Malignant lymphoma $\cdot$ Pancreatic cancer $\cdot$ Chronic pancreatitis . Autoimmune pancreatitis

\section{Introduction}

Malignant lymphoma of the pancreas is a rare solid tumour categorised as one of the non-epithelial tumours. Primary pancreatic lymphoma arising from lymphoid elements in the pancreas itself is extremely rare, and it is defined by following clinical criteria [1]:

(1) No evidence of palpable superficial lymphadenopathy,

(2) No enlargement of mediastinal nodes,

(3) Normal leukocyte count,

(4) At surgery, the pancreatic mass predominates, with involved lymph nodes confined to the peripancreatic region,

(5) No hepatic or splenic involvement.

Extranodal lymphoma is classified as secondary if there is involvement of lymph nodes except for those of an adjacent primary organ or with more than one extra nodal site. Lymphomatous involvement of the pancreas is usually of the non-Hodgkin's or B-cell type. Overall, secondary involvement of the pancreas with lymphoma is more common than primary pancreatic lymphoma, which accounts for less than $1 \%$ of pancreatic tumours [1-4] and less than $2 \%$ of extra-nodal lymphoma $[5,6]$. On the other hand, secondary lymphoma is much more common and can be seen in up to $30 \%$ of patients presenting with widespread lymphoma [1]. However, predominant pancreatic involvement is rare. Diffuse large B-cell is the most common type of nonHodgkin's lymphoma (NHL), and more than $50 \%$ of patients have some extra-nodal lesions, comprising less than $0.6-2.1 \%$ of pancreatic NHL $[7,8]$. Secondary malignant lymphomas were found in $3 \%$ of autopsy cases with 
pancreatic secondary tumours [9], usually appearing as large and bulky masses.

The incidence of pancreatic involvement is as high as $5 \%$ in human immunodeficiency virus (HIV) patients. The gastrointestinal tract is the most commonly affected extranodal site in AIDS-related NHL [10-12].

\section{Clinical manifestations of pancreatic malignant lymphoma}

Primary pancreatic lymphoma appears as variable histopathological subtypes, such as follicular lymphoma, lymphoma of mucosa-associated lymphoid tissue, diffuse large B-cell lymphoma and T-cell lymphoma. It affects patients in the 5th or 6th decade of life and has a slight male predominance. Clinical symptoms are generally nonspecific; abdominal pain, abdominal mass and weight loss are the most common symptoms, followed by jaundice, nausea, vomiting, diarrhoea, pancreatitis and bowel obstruction [13, 14]. Jaundice is sometimes observed because the head of the pancreas is the most common location (more than $80 \%$ ) [14]. These findings are similar to those in secondary lymphoma with predominantly pancreatic involvement, although the classic symptoms of NHL, such as fever, chills and night sweats, are rarely seen in primary pancreatic lymphoma. The incessant pain typically encountered with pancreatic adenocarcinoma (PAC) is rare [13]. Therefore, an important clinical aspect differentiating PAC is the clinical manifestation of abdominal pain and palpable mass without jaundice. Primary pancreatic lymphoma can also be associated with immunodeficiency syndromes. There are reported cases of pancreatic lymphoma following solid-organ transplantation or patients infected with HIV [12, 15].

Surgical resection does not play a role in the treatment of pancreatic lymphoma, because chemotherapy is effective in most of the patients. However, pancreatic lymphoma is frequently treated with surgical resection because of the difficulty of differentiating it from PAC [16, 17]. Although biopsy is one of the most useful methods for accurate diagnosis $[17,18]$, preoperative diagnosis by non-invasive methods is of the utmost importance.

\section{MR technique}

A comprehensive MR protocol to fully evaluate the pancreatic parenchyma and the pancreaticobiliary ductal system at our institution consists of the following sequences: T1weighted gradient-echo (GRE); T2-weighted axial and coronal sequences, usually fast spin-echo (FSE) or a variant of
FSE; diffusion-weighted imaging (DWI); 2D and 3D MR cholangiopancreatography (MRCP); and T1-weighted 3D GRE before and after contrast [19].

Fat-suppressed T1-weighted images (FST1WI) play an important role in detecting lesions because normal pancreatic parenchyma has either a homogeneous or cobblestone appearance and appears as hyperintense signal areas on FST1WI [20]. Lesions within the pancreas are observed as hypointense signal areas compared with the surrounding pancreatic parenchyma. In addition, the haemorrhagic component in the lesion shows up as hyperintense signal areas.

T2-weighted images (T2WI) clearly demonstrate the internal appearance of the lesion. Presence of necrosis and cystic components is rare in lymphoma but can be seen in other tumours [21]. Thin-slice ultrafast T2WI by a partial Fourier method provides images that clearly demonstrate the association between the lesion and the main pancreatic duct (MPD).

DWI, reflecting changes in water mobility, offers high detectability of the lesions. The respiratory-triggered technique is more effective for providing better image quality, signal intensity characteristics and apparent diffusion coefficient (ADC) measurement than the free-breath technique [22]. ADC values are useful for differentiating between benign and malignant tumours in most studies. However, radiologist should know that $\mathrm{ADC}$ values in the pancreas head are lower than those in the tail [23] and it is sometimes difficult to differentiate malignant tumours from inflammatory lesions [24].

MRCP, provided by heavily T2WI, is a useful and noninvasive method to assess the MPD and the biliary tree. Freebreathing MRCP obtained by the 3D FSE sequence improves the diagnostic performance, image quality and visibility of the pancreatic duct compared with conventional breath-hold 2D MRCP [25].

Obtaining a dynamic contrast-enhanced MR image (DCEMRI) using extravascular gadolinium contrast agents is useful to assess the vascularity of the tumour. FST1WI using a chemical-shift-selective fat-saturation method is recommended to eliminate paradoxical negative enhancement [26]. Overall, the signal-to-noise ratio of 3-T MRI is superior to that of $1.5 \mathrm{~T}$, and high-resolution, thin-slice images can be obtained by 3DT1WI sequences using the partial-Fourier method [19].

\section{CT and MR features of pancreatic lymphoma}

In previous reports, radiologic findings of secondary lymphoma with predominant pancreatic involvement were similar to those of primary pancreatic lymphoma including nodular, diffuse and multi-nodular types [14, 27, 28].

On CT imaging with intravenous contrast, most lesions are shown as well defined, sometimes bulky and infiltrating, 
Table 1 Comparison of MR findings among malignant lymphoma, autoimmune pancreatitis and pancreatic adenocarcinoma

\begin{tabular}{|c|c|c|c|}
\hline Finding & Lymphoma & AIP & PAC \\
\hline Speckled hyperintensity on FST1WI ${ }^{\mathrm{a}}$ & None & Sometimes & Rare \\
\hline Target appearance on $\mathrm{T}_{2} \mathrm{WI}^{\mathrm{b}}$ & Rare & Rare & Frequent ${ }^{\star}$ \\
\hline Duct-penetrating sign (in focal lesions) & Rare & Sometimes & Rare \\
\hline Marked upstream MPD dilatation & Sometimes & Rare & Frequent ${ }^{*}$ \\
\hline Speckled enhancement on PP DCE-MRI ${ }^{\mathrm{c}}$ & None & Frequent & Rare \\
\hline Homogeneous enhancement on EP DCE-MRI & Sometimes & Common & Rare \\
\hline Target appearance on PP or EP DCE-MRI ${ }^{\mathrm{b}}$ & Rare & Rare & Common $^{\ddagger}$ \\
\hline Without delayed enhancement & Frequent* & Rare & Rare \\
\hline Diffuse enlargement (sausage appearance) & Sometimes & Sometimes & Rare \\
\hline Capsule-like rim (in diffuse lesions) & Rare & Frequent $^{*}$ & None \\
\hline Multi-focal lesions & Sometimes & Sometimes & None \\
\hline Vascular involvement without obstruction & Sometimes $^{\dagger}$ & Sometimes & Rare \\
\hline Lymphadenopathy below the level of the left renal vein & Sometimes $^{\dagger}$ & Rare & Rare \\
\hline Other organ involvement & None (primary)/common (secondary) & Common & Sometimes (metastasis) \\
\hline
\end{tabular}

AIP autoimmune pancreatitis, $P A C$ pancreatic adenocarcinoma, FST1WI fat-suppressed T1-weighted image, $T 2 W I$ T2-weighted image, $M P D$ main pancreatic duct, $D C E-M R I$ dynamic contrast-enhanced magnetic resonance imaging, $P P$ pancreatic phase, $E P$ equilibrium phase

${ }^{a}$ Speckled hyperintensity is defined as a hypointense or isointense lesion including speckled or dotted hyperintense areas

${ }^{\mathrm{b}}$ Target sign is defined as a hyperintense lesion surrounding a more hyperintense focal area on fat-suppressed T2WI or a hypointense to hyperintense lesion surrounding a more hypointense area on DCE-MRI

${ }^{\mathrm{c}}$ Speckled enhancement is defined as hypointense or isointense areas including speckled or dotted enhanced areas on DCE-MRI

* Specific finding in comparison between malignant lymphoma and AIP

${ }^{\dagger}$ Specific finding in comparison between malignant lymphoma and PAC

${ }^{\star}$ Specific finding in comparison between AIP and PAC

homogeneous low-attenuation masses relative to the enhancing pancreatic parenchyma with only mild enhancement [13]. However, lesions that are less well circumscribed may wrongly be misdiagnosed as pancreatic cancer and result in surgical intervention.

Less commonly, pancreatic lymphoma may present as diffuse enlargement of the gland with an infiltrative tumour that could mimic acute pancreatitis. It is important to note that typical features of acute pancreatitis, including peripancreatic fat stranding and peripancreatic inflammation, are usually minimal if even present. Peripancreatic fluid collections and fat necrosis are typically absent in infiltrating pancreatic lymphoma. To our knowledge, pancreatic ductal disconnection and disruption seen with severe acute pancreatitis has not been reported in lymphoma. Vascular encasement or invasion is rarely seen with pancreatic lymphoma [13]. Although vessels may be stretched because of a mass effect, irregularity and caliber changes due to tumour invasion are generally absent. Intravascular lymphomatosis is a rare type of malignant lymphoma, whose pathologic findings have been reported [29].

MRI is an excellent modality for detecting and diagnosing pancreatic lesions as well as assessing the extent of involvement, since the tissue contrast is far superior compared with CT. In general, pancreatic lymphomas appear as homogeneous, low-signal-intensity, focal nodular areas on T1WI, with variable, low or high signal intensity on T2WI, and a generally circumscribed, less-enhanced area relative to surrounding parenchyma on DCE-MRI [13, 28]. Unlike CT, MRI shows a slightly heterogeneous character of the lesion, especially on T2WI. Islet cell tumours, although circumscribed, tend to show more hyperintensity than lymphoma on $\mathrm{T} 2 \mathrm{~W}$ images. There are no reports describing pancreatic lymphoma on DWI; however, whole-body DWI is already playing an important role in detecting and staging in lymphoma patients.

Following administration of intravenous gadoliniumbased contrast agents, lymphomatous deposits enhance homogeneously, but to a lesser degree than normal parenchyma. Some lymphomatous lesions can appear mildly inhomogeneous on MRI. On the other hand adenocarcinoma of the pancreas tends to enhance less because of the desmoplastic content and is typically inhomogeneous post gadolinium.

It is important to differentiate pancreatic lymphoma from its mimickers, such as autoimmune pancreatitis (AIP) and PAC, because of the difference in their treatment and prognosis. Based on the previous reports, useful MR findings in the differentiation between them are summarised in Table 1 [13, 28, 30-32]. Since there are few reports describing MR imaging findings of pancreatic malignant lymphoma, we 

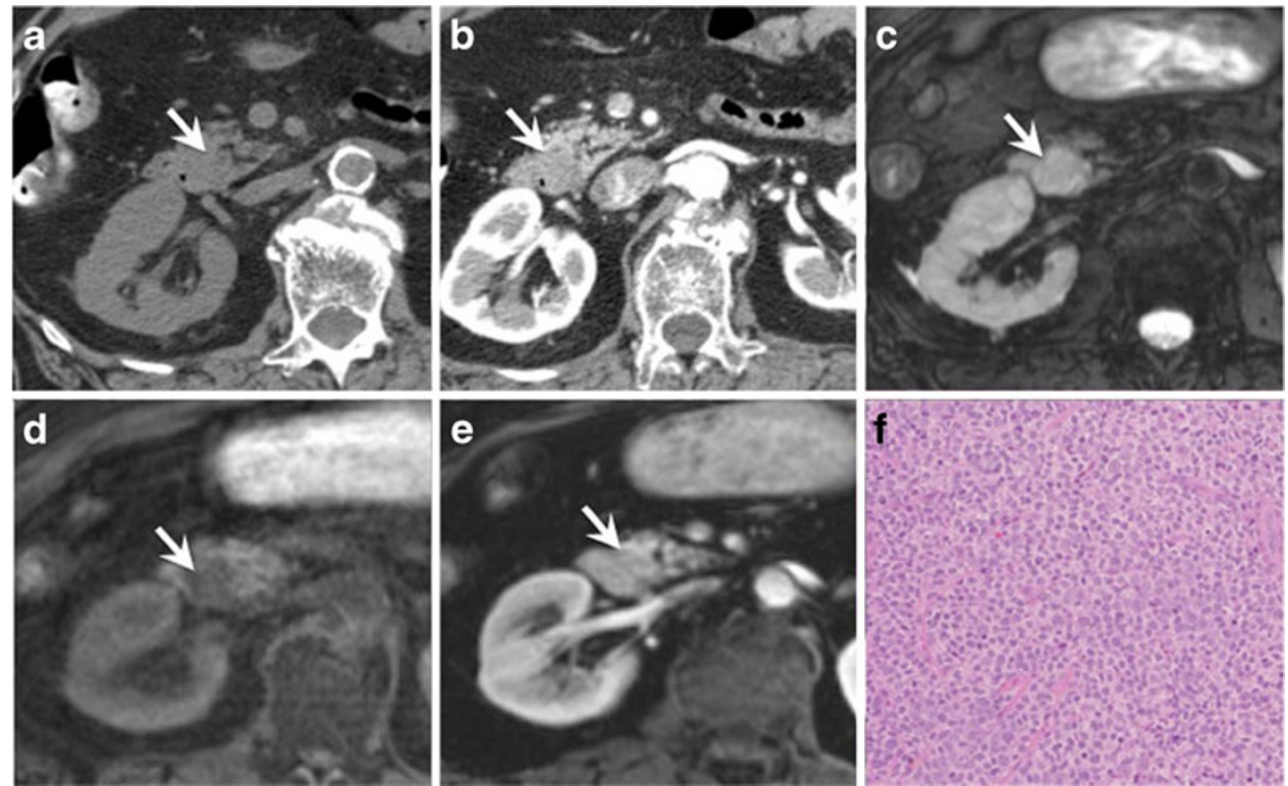

Fig. 1 An 82-year-old woman with primary pancreatic lymphoma, biopsy-proven diffuse large B-cell lymphoma. a Precontrast CT shows ill-defined pancreas head mass (white arrow). b Pancreatic phase dynamic contrast-enhanced CT shows round-shaped and slightly low density mass (white arrow). c Fat-suppressed T2-weighted MR image shows a circumscribed homogeneously hyperintense mass in the pancreatic head (white arrow). d Fat-suppressed T1-weighted MR image
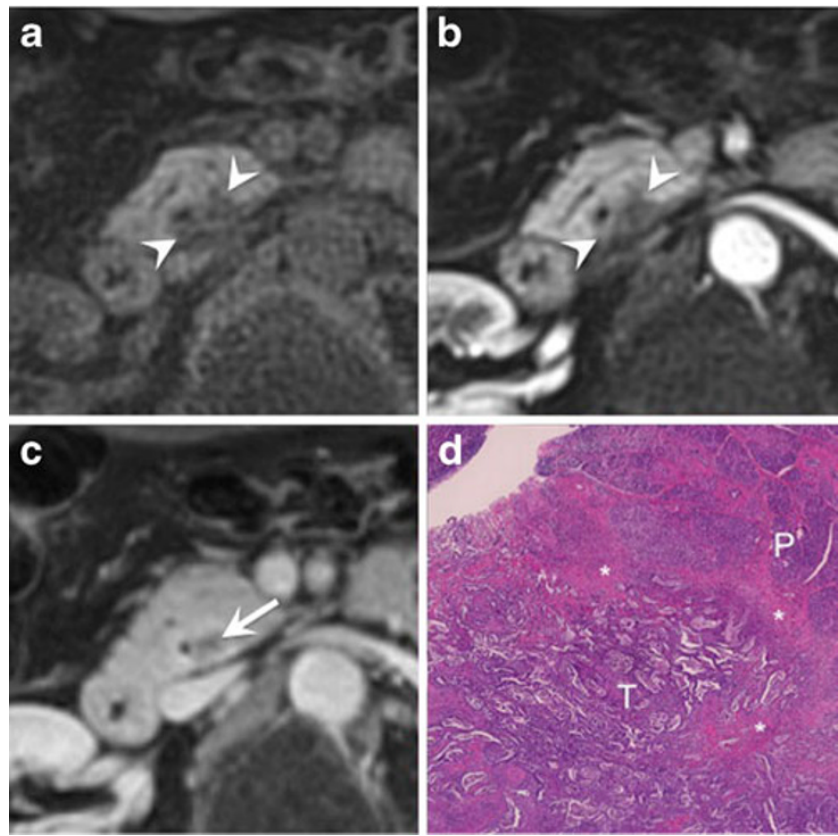

Fig. 2 A 58-year-old man with pancreas head adenocarcinoma. a Fatsaturated T1-weighted image shows a hypointense pancreas head mass (white arrowheads). b Pancreatic phase dynamic contrast-enhanced MR image shows a hypointense mass with irregular border (white arrowheads). c Equilibrium phase of dynamic contrast-enhanced MR image shows heterogeneous delayed enhancement of the mass with a poorly enhancing area (white arrow). d Haematoxylin-eosin-stained histologic section (original magnification $\times 15$ ) shows well-differentiated tumour cells (T) with fibrous stroma $\left(^{*}\right)$. $\mathrm{P}$, adjacent pancreatic parenchyma shows a hypointense mass in the pancreatic head (white arrow) relative to adjoining normal pancreatic parenchyma. e Pancreatic phase of dynamic contrast-enhanced MR image shows a circumscribed poorly enhanced mass (white arrow) in the pancreatic head with normally enhanced adjacent pancreatic parenchyma. f Haematoxylin-eosinstained histologic section (original magnification $\times 200$ ) shows the tumour composed of atypical large lymphoid cells with large nucleus

have demonstrated the MR features and varied imaging presentations of this disease to alert the interpreting radiologist and prevent misdiagnosis and inadvertent surgery.

Well-circumscribed nodular type with solitary pancreatic mass

Most of the patients in previous case reports were categorised into this type. In the previous studies comprising a large number of cases, 4 of $6(66.7 \%)$ cases of primary pancreatic lymphoma and 9 of $12(75.0 \%)$ cases of secondary pancreatic lymphoma were classified into this type [27,33]. This type of presentation is the most commonly encountered and is shown as a homogeneous, generally circumscribed, low-signalintensity area on T1WI, homogeneous or slightly heterogeneous on T2WI, and a distinctly hypovascular area on DCEMRI compared with surrounding normal parenchyma (Fig. 1). Lymphoma on the whole tends to be better marginated than PAC, with a clearer demarcation of tumour and normal tissue. On rare occasions, the heterogeneous appearance and poor definition on both T1WI and T2WI can lead to misdiagnosis of PAC (Fig. 2) [34, 35]. There are some useful findings to differentiate lymphoma from PAC, as follows: (1) A bulky homogeneous mass without cystic change, necrosis and alteration of MPD; (2) the envelopment of adjacent vessels without evidence of obstruction; (3) the presence of lymphadenopathy below the level of the left renal vein if present is typically in 

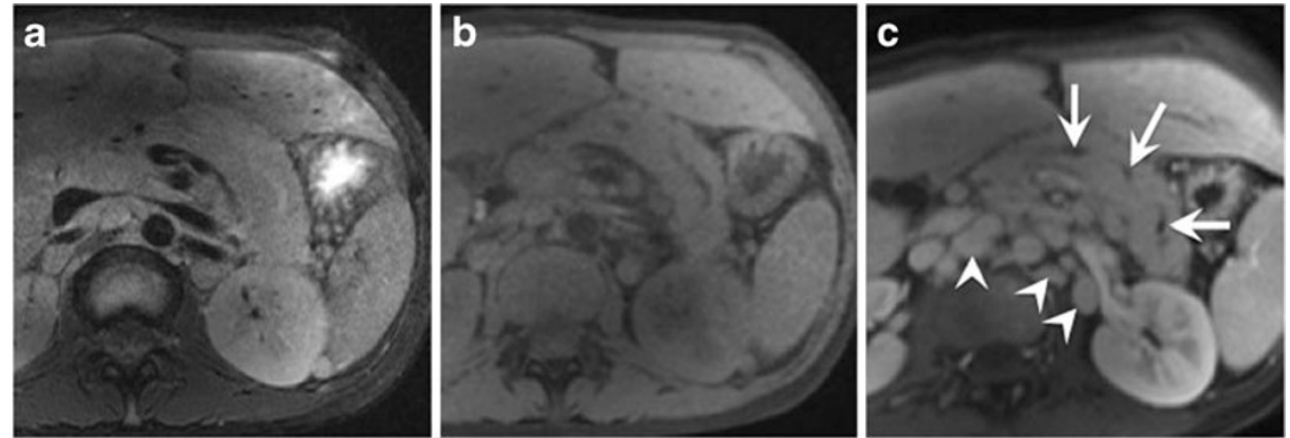

Fig. 3 A 19-year-old woman with biopsy-proven primary Hodgkin's lymphoma involving the pancreas. a Fat-suppressed T2-weighted image shows diffuse pancreatic enlargement and homogeneously hyperintense pancreatic parenchyma comparable to spleen. b Fatsuppressed T1-weighted image shows homogeneously hypointense

the peripancreatic region [13]. However, CT-guided biopsy or endoscopic ultrasound-guided fine-needle aspiration (EUSFNA) may sometimes be needed for accurate diagnosis.

\section{Diffuse type with pancreatic enlargement}

In the previous studies, 1 of $6(16.7 \%)$ cases of primary pancreatic lymphoma and 2 of $12(16.7 \%)$ cases of secondary pancreatic lymphoma were classified into this category $[27,33]$. Thus, diffuse pancreatic enlargement with mild MPD dilatation is another presentation of pancreatic lymphoma (Fig. 3). While a distinct nodular mass may not be appreciated, a subtle clue to the diagnosis may simply be upstream MPD dilatation in some cases (Fig. 4). Signal intensity of the pancreas that is involved diffusely with lymphoma is generally diffusely decreased on T1WI and diffusely increased on T2WI. The findings of this type may closely mimic the imaging findings of acute pancreatitis [36, 37]. The key to diagnosis is that peripancreatic inflammation pancreatic parenchyma. c Portal phase of dynamic contrast-enhanced dynamic MR image shows poorly enhanced pancreas. Focal main pancreatic duct dilatation (white arrow) and multiple adjacent lymph nodes (white arrowheads) are seen. Note lack of vascular invasion

is typically minimal or completely absent without associated fluid collections or extension of inflammation into the retroperitoneal spaces/mesenteric root. When enlarged peripancreatic or retroperitoneal lymph nodes are present, exclusion of acute pancreatitis is much easier.

Peripheral lymphomatous involvement, mimicking autoimmune pancreatitis

In rare cases (the frequency of this type is unclear), the poorly defined margin of a focally enlarged pancreas and a poorly defined capsule-like rim may be noted in lymphomatoid involvement (Fig. 5). While this is a typical finding of AIP (Fig. 6) [38], Ishigami et al. [28] reported that high signal intensity on T2WI and absence of delayed enhancement might be helpful for differentiating pancreatic lymphoma from AIP. The rim tends to be fairly hypointense on both T1WI and T2WI and is typically of uniform thickness in autoimmune pancreatitis. Lymphadenopathy can be

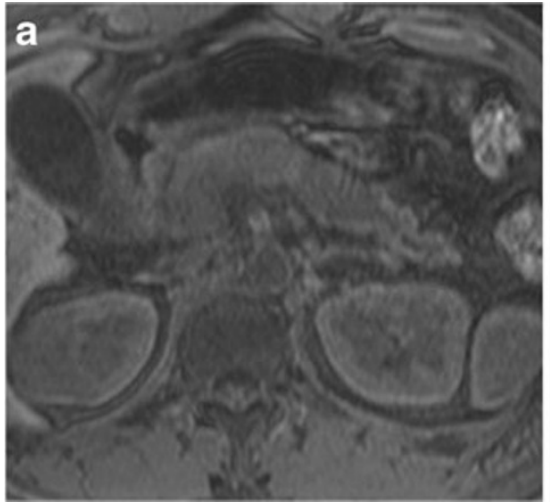

Fig. 4 A 56-year-old man with secondary pancreatic lymphoma. a Fat-suppressed T1-weighted image shows diffuse pancreatic swelling and homogeneously hypointense pancreas. Atrophic pancreatic and residual normal pancreatic parenchyma of the tail are seen. $\mathbf{b}$ Pancreatic phase of dynamic contrast-enhanced MR image shows

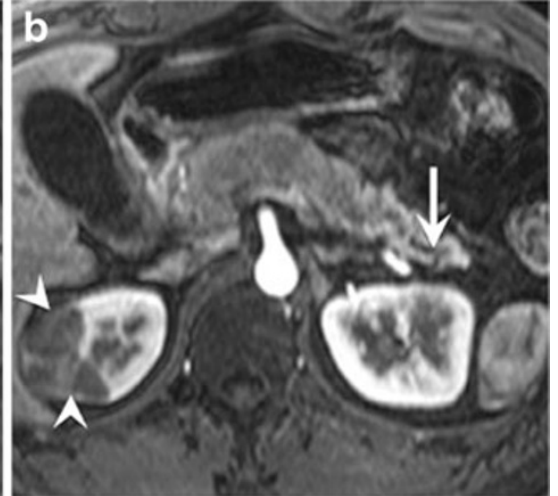

poorly enhanced pancreas head and body. Distal main pancreatic duct dilatation (white arrow) and enhanced normal pancreatic parenchyma are seen. Poorly enhanced right renal lesion (white arrowheads) is also seen 

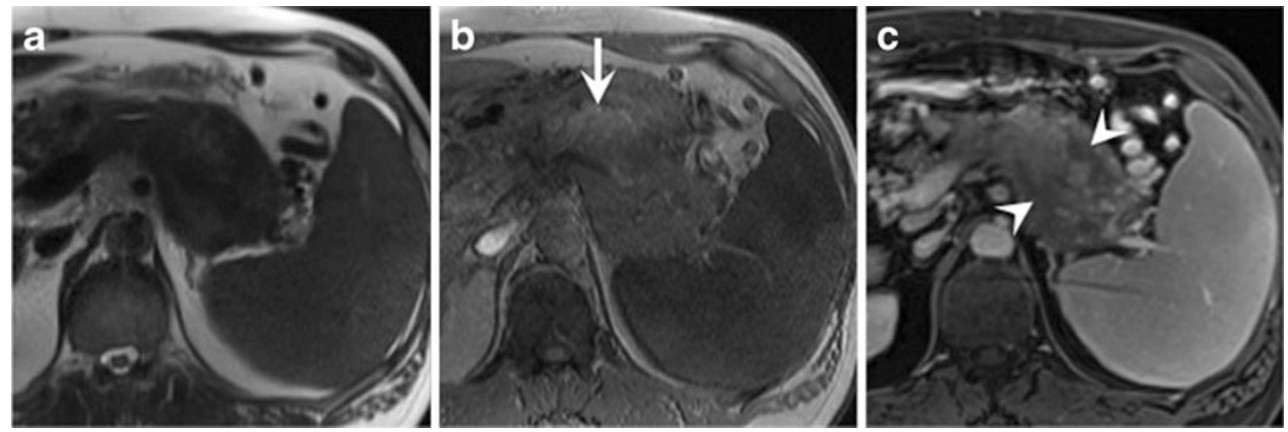

Fig. 5 A 57-year-old man with secondary pancreatic lymphoma. a Axial T2-weighted image shows diffuse pancreatic swelling with pancreas parenchyma homogeneously hypointense relative to spleen. b Axial T1-weighted image shows that the majority of the pancreas is heterogeneously hypointense with a hyperintense area (white arrow), representing residual normal pancreatic parenchyma, in the centre of the lesion. c Portal phase of dynamic contrast-enhanced MR image shows poorly enhanced peripheral area mimicking capsule-like rim (white arrowheads). The rim however does not have the characteristic appearance of the typical hypointense, uniform thickness rim in autoimmune pancreatitis

it. EUS-FNA may be required to reach an accurate diagnosis in cases with confusing imaging findings.

seen in autoimmune pancreatitis and is not infrequent, confounding the diagnosis [39]. On the other hand, characteristic extra-pancreatic lesions, such as bile duct, renal and retroperitoneal lesions, frequently seen in AIP, are useful findings for differentiation [39]. Challenging with corticosteroid therapy is not recommended because pancreatic swelling in both lymphoma and AIP can be improved by
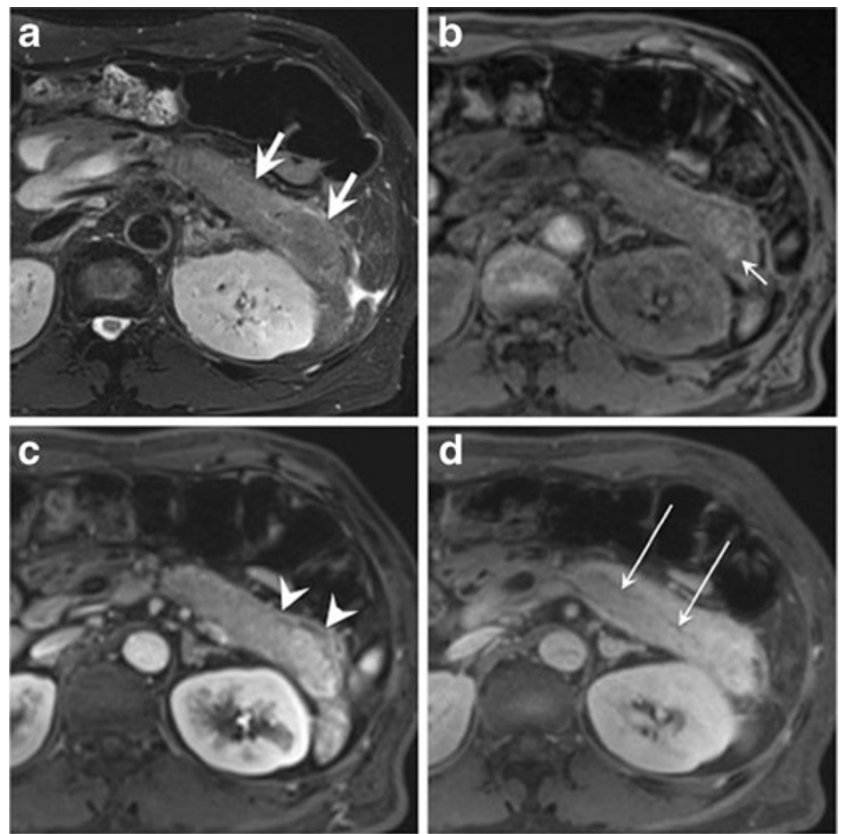

Fig. 6 A 57-year-old man with autoimmune pancreatitis. a Fatsuppressed T2-weighted image shows sausage-like swelling of the pancreas body and tail, which is seen as hyperintensity area (white arrows). $\mathbf{b}$ Fat-suppressed T1-weighted image shows slightly hyperintense area in the lesion (white small arrow). c Pancreatic phase of dynamic contrastenhanced MR image shows capsule-like enhancement surrounding the lesion (white arrowheads). d Equilibrium phase of dynamic contrastenhanced MR image shows homogeneously enhanced lesion and irregular narrowing of the main pancreatic duct (white long arrows)

\section{Muti-nodular type}

Multi-nodular type was seen in 2 of $8(25 \%)$ patients with primary and secondary pancreatic lymphoma and 1 of 12 $(8.3 \%)$ with secondary pancreatic lymphoma [27, 28]. Multiple nodular lesions in the pancreas are seen in this type and every nodular lesion appears quite discrete with similar findings as seen with the well-circumscribed tumoral type (Fig. 7). This type can at times mimic the diffuse type if there are confluent or aggregated tumours. MPD dilatation however is not typical. Differential diagnosis is multiple metastases from hypovascular tumours, such as gallbladder, lung and ovarian cancer [40], and multifocal involvement with AIP (Fig. 8) [41]. Necrosis and cystic change are more likely to be seen in the hypointense/isointense lesion including speckled/dotted
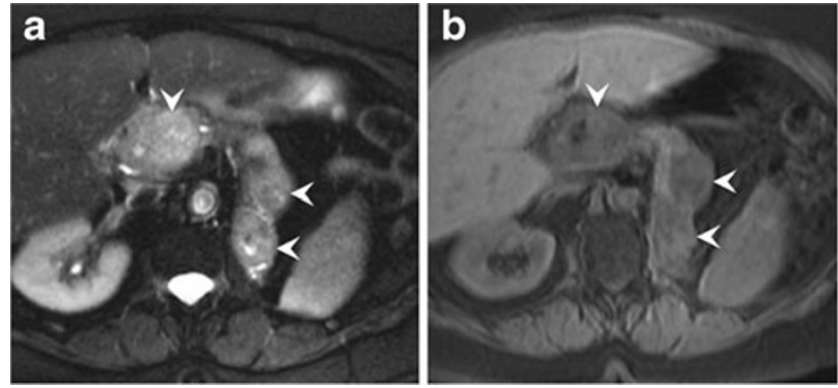

Fig. 7 A 77-year-old woman with multifocal pancreatic lymphoma. a Axial fat-suppressed T2-weighted image shows multiple heterogeneously hyperintense masses in the pancreas head, body and tail (white arrowheads). b Axial fat-suppressed T1-weighted image shows multiple heterogeneous hypointense masses in the pancreas head, body and tail (white arrowheads) metastases of epithelial primary tumours. On the other hand, a 

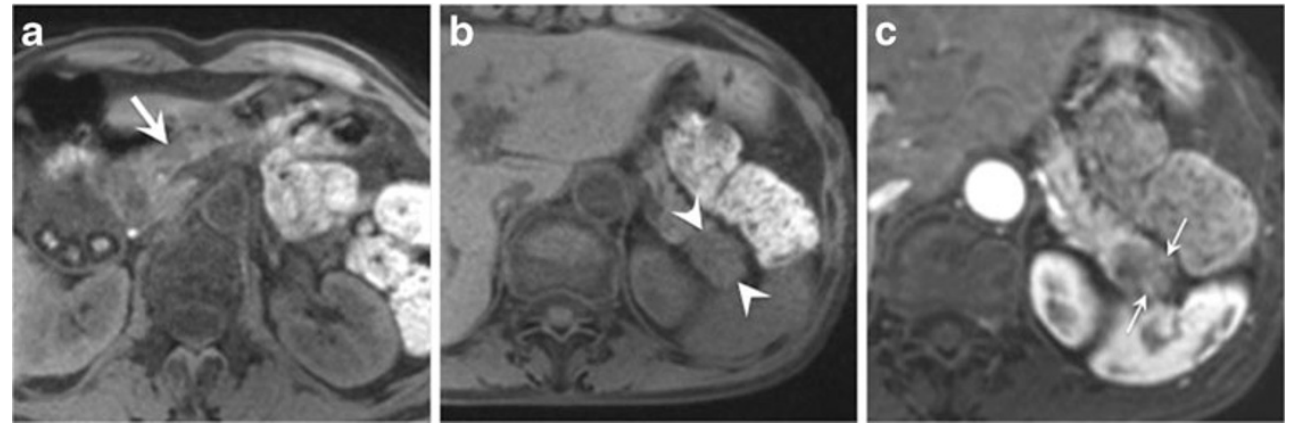

Fig. 8 A 72-year-old woman with multifocal autoimmune pancreatitis. a Fat-suppressed T1-weighted image shows small hypointense area in the pancreas head (white arrow). b Fat-suppressed T1-weighted image (cranial slice of a) shows a hypointense mass of the pancreas tail (white arrowheads). c Pancreatic phase of dynamic contrast-enhanced MR image shows speckled enhancement in the pancreas tail lesion (small arrows)

usual. CT-guided biopsy is recommended for retroperitoneal lesions for accurate and quick diagnosis if primary lesions are not found. hanced areas on DCE-MRI (speckled enhancement) are useful findings to diagnose focal AIP [30]. Biopsy is sometimes necessary for accurate diagnosis when there is no typical clinical and radiologic finding.

Invasion from an adjacent peri-pancreatic lymphomatous lesion

Malignant lymphoma usually appears as a well-circumscribed bulky mass, but can rarely present as invasive tumour [42]. In this situation, an extra-pancreatic lymphoma may directly extend or infiltrate into the adjacent pancreatic parenchyma, and the pancreatic lesion is recognised as a secondary malignant lymphoma. The signal of pancreatic parenchyma is focally and heterogeneously decreased on FST1WI and increased on T2WI (Fig. 9). Under these circumstances, detecting the primary location may become challenging; however, it is noted that invasion of the pancreas is fairly common with contiguous duodenal and other retroperitoneal lymphomas. Associated lymphadenopathy elsewhere in the abdomen is also fairly

\section{Conclusion}

MRI findings of primary and secondary lymphoma may at times not be sufficiently specific to render a diagnosis, especially when pancreatic involvement is the first or only finding. Rather, other parameters must be considered, including clinical presentation and relevant imaging studies.

Radiologists should, however, be well versed in these varied imaging manifestations, as well as the characteristic findings of pancreatic lymphoma and its mimickers, to avoid misdiagnosis and unnecessary surgery. In a few cases, the findings of pancreatic lymphoma may be confusing and endoscopic biopsy should be suggested to arrive at a definitive diagnosis.

Acknowledgments We thank Dr. Hiroyoshi Ota for pathological instructions.
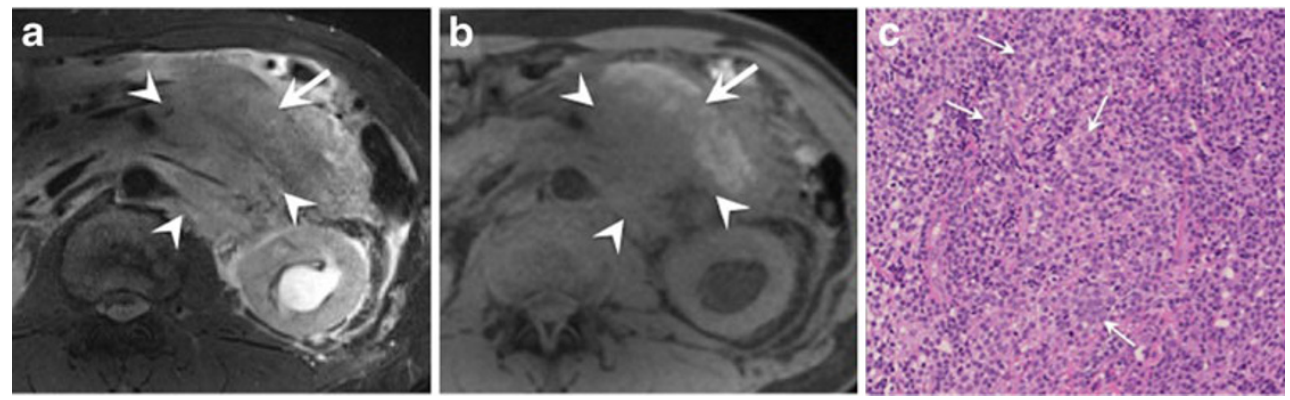

Fig. 9 A 56-year-old man with secondary pancreatic lymphoma, biopsy-proven follicular lymphoma. a Axial fat-suppressed T2-weighted image shows invasively spreading hyperintense retropancreatic mass (white arrowheads) and heterogeneously hyperintense pancreas (white arrow). b Axial fat-suppressed T1-weighted image shows hypointense retropancreatic mass (white arrowheads) pushes pancreatic parenchyma anteriorly. Involved pancreatic parenchyma is seen as a heterogeneously hypointense area (white arrow). c Haematoxylin-eosin-stained histologic section (original magnification, $\times 200$ ) shows the tumour with atypical large lymphoid cells aggregated focally (white small arrows). The tumour cells are stained positive for CD10 antigen and bcl2 gene by immunohistochemical analysis (not shown) 
Open Access This article is distributed under the terms of the Creative Commons Attribution License which permits any use, distribution, and reproduction in any medium, provided the original author(s) and the source are credited.

\section{References}

1. Behrns KE, Sarr MG, Strickler JG (1994) Pancreatic lymphoma: is it a surgical disease? Pancreas 9(5):662-667

2. Weingrad DN, Decosse JJ, Sherlock P, Straus D, Lieberman PH, Filippa DA (1982) Primary gastrointestinal lymphoma: a 30-year review. Cancer 49(6):1258-1265

3. Tanaka T, Matsugu Y, Koide K et al (1996) Malignant lymphoma of the pancreas. Dig Dis Sci 41(2):402-404

4. Isaacson PG (1999) Gastrointestinal lymphomas of T- and B-cell types. Mod Pathol 12(2):151-158

5. Teefey SA, Stephens DH, Sheedy PF 2nd (1986) CT appearance of primary pancreatic lymphoma. Gastrointest Radiol 11(1):41-43. doi:10.1007/BF02035029

6. Tuchek JM, De Jong SA, Pickleman J (1993) Diagnosis, surgical intervention, and prognosis of primary pancreatic lymphoma. Am Surg 59(8):513-518

7. Freeman C, Berg JW, Cutler SJ (1972) Occurrence and prognosis of extranodal lymphomas. Cancer 29(1):252-260

8. Takahashi H, Tomita N, Yokoyama M et al (2012) Prognostic impact of extranodal involvement in diffuse large B-cell lymphoma in the rituximab era. Cancer 118(17):4166-4172. doi:10.1002/cncr.27381

9. Nakamura E, Shimizu M, Itoh T, Manabe T (2001) Secondary tumors of the pancreas: clinicopathological study of 103 autopsy cases of Japanese patients. Pathol Int 51(9):686-690

10. Dodd GD 3rd, Greenler DP, Confer SR (1992) Thoracic and abdominal manifestations of lymphoma occurring in the immunocompromised patient. Radiol Clin N Am 30(3):597-610

11. Radin DR, Esplin JA, Levine AM, Ralls PW (1993) AIDS-related non-Hodgkin's lymphoma: abdominal CT findings in 112 patients. AJR Am J Roentgenol 160(5):1133-1139

12. Jones WF, Sheikh MY, McClave SA (1997) AIDS-related non-Hodgkin's lymphoma of the pancreas. Am J Gastroenterol 92(2):335-338

13. Merkle EM, Bender GN, Brambs HJ (2000) Imaging findings in pancreatic lymphoma: differential aspects. AJR Am J Roentgenol 174(3):671-675

14. Saif MW (2006) Primary pancreatic lymphomas. JOP 7(3):262-273

15. Cario E, Runzi M, Metz K, Layer P, Goebell H (1997) Diagnostic dilemma in pancreatic lymphoma. Case report and review. Int $\mathrm{J}$ Pancreatol 22(1):67-71. doi:10.1007/BF02803907

16. Du X, Zhao Y, Zhang T et al (2011) Primary pancreatic lymphoma: a clinical quandary of diagnosis and treatment. Pancreas 40(1):3036. doi:10.1097/MPA.0b013e3181e6e3e5

17. Arcari A, Anselmi E, Bernuzzi P et al (2005) Primary pancreatic lymphoma. Report of five cases. Haematologica 90(2):ECR09

18. Nayer H, Weir EG, Sheth S, Ali SZ (2004) Primary pancreatic lymphomas: a cytopathologic analysis of a rare malignancy. Cancer 102(5):315-321. doi:10.1002/cncr.20488

19. Sandrasegaran K, Lin C, Akisik FM, Tann M (2010) State-of-the-art pancreatic MRI. AJR Am J Roentgenol 195(1):42-53. doi:10.2214/ AJR. 10.4421

20. Gabata T, Matsui O, Kadoya M et al (1994) Small pancreatic adenocarcinomas: efficacy of MR imaging with fat suppression and gadolinium enhancement. Radiology 193(3):683-688
21. Mergo PJ, Helmberger TK, Buetow PC, Helmberger RC, Ros PR (1997) Pancreatic neoplasms: MR imaging and pathologic correlation. Radiographics 17(2):281-301

22. Kartalis N, Loizou L, Edsborg N, Segersvard R, Albiin N (2012) Optimising diffusion-weighted MR imaging for demonstrating pancreatic cancer: a comparison of respiratory-triggered, free-breathing and breath-hold techniques. Eur Radiol 22(10):2186-2192. doi:10.1007/s00330-012-2469-3

23. Schoennagel BP, Habermann CR, Roesch M et al (2011) Diffusion-weighted imaging of the healthy pancreas: apparent diffusion coefficient values of the normal head, body, and tail calculated from different sets of b-values. J Magn Reson Imaging 34(4):861-865. doi:10.1002/jmri.22743

24. Fukukura Y, Takumi K, Kamimura K et al (2012) Pancreatic adenocarcinoma: variability of diffusion-weighted MR imaging findings. Radiology 263(3):732-740. doi:10.1148/radiol.120111222

25. Kim JH, Hong SS, Eun HW, Han JK, Choi BI (2012) Clinical usefulness of free-breathing navigator-triggered 3D MRCP in non-cooperative patients: comparison with conventional breath-hold 2D MRCP. Eur J Radiol 81(4):e513-e518. doi:10.1016/j.ejrad.2011.06.004

26. Mitchell DG, Stolpen AH, Siegelman ES, Bolinger L, Outwater EK (1996) Fatty tissue on opposed-phase MR images: paradoxical suppression of signal intensity by paramagnetic contrast agents. Radiology 198(2):351-357

27. Cappell MS, Yao F, Cho KC, Axiotis CA (1989) Lymphoma predominantly involving the pancreas. Dig Dis Sci 34(6):942-947

28. Ishigami K, Tajima T, Nishie A et al (2010) MRI findings of pancreatic lymphoma and autoimmune pancreatitis: a comparative study. Eur J Radiol 74(3):e22-e28. doi:10.1016/j.ejrad.2009.03.022

29. Lui PC, Wong GK, Poon WS, Tse GM (2003) Intravascular lymphomatosis. J Clin Pathol 56(6):468-470

30. Sugiyama Y, Fujinaga Y, Kadoya M et al (2012) Characteristic magnetic resonance features of focal autoimmune pancreatitis useful for differentiation from pancreatic cancer. Jpn J Radiol 30(4):296-309. doi:10.1007/s11604-011-0047-2

31. Muhi A, Ichikawa T, Motosugi U et al (2012) Mass-forming autoimmune pancreatitis and pancreatic carcinoma: differential diagnosis on the basis of computed tomography and magnetic resonance cholangiopancreatography, and diffusion-weighted imaging findings. J Magn Reson Imaging 35(4):827-836. doi:10.1002/jmri.22881

32. Hur BY, Lee JM, Lee JE et al (2012) Magnetic resonance imaging findings of the mass-forming type of autoimmune pancreatitis: comparison with pancreatic adenocarcinoma. J Magn Reson Imaging 36(1):188-197. doi:10.1002/jmri.23609

33. Lin H, Li SD, Hu XG, Li ZS (2006) Primary pancreatic lymphoma: report of six cases. World J Gastroenterol 12(31):5064-5067

34. Wang YJ, Jeng CM, Wang YC, Chang PP, Wang TH (2006) Primary pancreatic Burkitt's lymphoma mimicking carcinoma with obstructive jaundice and very high CA19-9. Eur J Gastroenterol Hepatol 18(5):537-540

35. Sata N, Kurogochi A, Endo K, Shimura K, Koizumi M, Nagai H (2007) Follicular lymphoma of the pancreas: a case report and proposed new strategies for diagnosis and surgery of benign or low-grade malignant lesions of the head of the pancreas. JOP 8(1):44-49

36. Van Beers B, Lalonde L, Soyer P et al (1993) Dynamic CT in pancreatic lymphoma. J Comput Assist Tomogr 17(1):94-97

37. Amodio J, Brodsky JE (2010) Pediatric Burkitt lymphoma presenting as acute pancreatitis: MRI characteristics. Pediatr Radiol 40(5):770-772. doi:10.1007/s00247-009-1475-3

38. Irie H, Honda H, Baba S et al (1998) Autoimmune pancreatitis: CT and MR characteristics. AJR Am J Roentgenol 170(5):1323-1327 
39. Fujinaga Y, Kadoya M, Kawa S et al (2010) Characteristic findings in images of extra-pancreatic lesions associated with autoimmune pancreatitis. Eur J Radiol 76(2):228-238. doi:10.1016/j.ejrad.2009.06.010

40. Reddy S, Edil BH, Cameron JL et al (2008) Pancreatic resection of isolated metastases from nonpancreatic primary cancers. Ann Surg Oncol 15(11):3199-3206. doi:10.1245/s10434-008-0140-7
41. Inoue D, Gabata T, Matsui O, Zen Y, Minato H (2006) Autoimmune pancreatitis with multifocal mass lesions. Radiat Med 24(8):587-591. doi:10.1007/s11604-006-0071-9

42. Ueda K, Nagayama Y, Narita K, Kusano M, Mernyei M, Kamiya M (2000) Pancreatic involvement by non-Hodgkin's lymphoma. J Hepatobiliary Pancreat Surg 7(6):610-613. doi:10.1007/s005340050243 